\title{
Dimensional Measurement of Complete-connective Network under the Condition of Particle's Fission and Growth at a Constant Rate*
}

\author{
Jinsong Wang, Beibei Hu
}

Department of management science and engineering, Beijing University of Technology, Beijing100124, P.R. China.

Email: xizixinxiang@163.com

Received 2012

\begin{abstract}
We construct a complete-connective regular network based on Self-replication Space and the structural principles of cantor set and Koch curve. A new definition of dimension is proposed in the paper, and we also investigate a simplified method to calculate the dimension of two regular networks. By the study results, we can get a extension: the formation of Euclidean space may be built by the process of the Big Bang's continuously growing at a constant rate of three times.
\end{abstract}

Keywords: Particle’S Fission; Regular Fractals; the Complete-Connective Network; Network Dimension

\section{Introduction}

The recent research shows that the fractal structure exists in massive networks, and there are different methods to calculate the fractal dimension of the network. Song et al. [1-3] adopted the Box counting method and Burning algorithm to measure the fractal dimensions of the networks; Kim et al. [4] also applied the Box counting method to the measurement of fractal dimensions of scale-free networks. However, building the network is still an under explored field which has not yet been adequately studied, especially by using a fractal set to identify the network and calculate its dimension.

In this paper, we attempt to make the further analysis on the construction of the network in the perspective of using the nodes of some regular fractals to build the networks. The study is based on the theory of self-replication algebraic space, and assumes the particle's fission grow at a constant rate. To build the network, we take the nodes from some regular fractals, such as the end point of cantor set and the apex of modified Koch curve. The study results suggest that the joint function of the correlation and connectivity among network nodes might influence the measurement of network dimension and space structure. Thus, this work contributes to re-measuring the dimension of the network from the perspective of the joint function of correlation and connectivity among network nodes.

\footnotetext{
*Project supported by the Major Program of the National Natural Science Foundation of China (Grant No. 71261026) National Key Technology R \& D Program of China (Grant No. 2012BAJ11B03)
}

\section{Problem Introduction}

If a particle $A$ generates $m$ particles after one fission, and get $m_{2}$ particles after twice fissions, $\cdots$, there will be $m^{n}$ articles after $n$ times of fissions, and these $m^{n}$ particles could form a space. In this space, the $m^{n}$ particles are taken as the nodes, which mean the salient points, and the network will form through the interaction among the $m$ particles. We use $C_{m^{n}}^{m}$ to describe the total correlation degree of the network, so the next step is to calculate the dimension of the constructed regular network space. To calculate the dimension from a new perspective of correlation and connectivity among network nodes, three hypotheses are introduced:

1) Self-replication Space: Particle $A$ generates $N$ particles after $n$ times fission. The particles are independent mutually in the proceeding of fission, at the same probability, and the correlation degrees among these particles are also the same with each other. Thus, the space formed by the $N$ particles is regarded as a Self-replication Space.

2) Network Connectivity: Regular fractals, such as the cantor set and the Koch curve, describe the fragmented and singular graphics which are self-similar with unlimited multi-levels. Following the principle of the cantor set, and by using its end point as the nodes, correlation is established among these nodes and then a completeconnective network is formed, as shown in Figure 1-((a)(b)). And in Figure 1-((c)(d)), the paper rebuilds the Koch curve based on its basic principles and then takes the apex of the modified Koch curve as the nodes. Finally, a complete-connective network is also formed 
through the correlation among these kinds of nodes.

3) Network transforms based on the cantor set and the Koch curve: It has been revealed that some of the regular fractals have a feature of the particle's fission at a constant rate. For example, the end points of the cantor set always grow at a constant fission rate of two times, and the apex of the modified Koch curve with the constant rate of four times. Therefore, on the premise of retaining the topological feature of fractals, the networks built in this paper are still based on the fundamentals of the cantor set and the Koch curve, and gain through the 2 or 3 times fission of particle $A$ which still exists during the fission. More specifically, we regard that all particles after fission own the homogeneous mutual correlation, so the particles $A, A_{1}, A_{2}$, or $A, A_{1}, A_{2}, A_{3}$ will be gained after the first fission of particle $A, \ldots$, continuously, the new particles will be generated after one more iteration, and finally there are $N$ particles after several fissions. These $N$ particles will form a complete-connective regular network. However, there are two additional assumed conditions: a) in order to get the network with self- similarity from $N$ particles, we first generate the network in accordance with an identically independently-distributed process with the same probability. (b)Additionally, when the $N$ particles are taken as the network nodes to build regular network, there is random distance between each nodes.

\section{A New Definition of Dimension and Mea- surement of Network Dimension}

Definition 1. We assume there are $N$ nodes, and they compose a network based on the interaction of the multiple nodes. The relevance $K$ is used to describe the
c
0

\begin{abstract}
$\Gamma$
\end{abstract}
e

lation degree of this network space, and its dimension $d$ is represented as:

$$
d=\lim _{n \rightarrow \infty} \frac{\ln K}{\ln N} .
$$

Theorem1. If particle $A$ generates $m$ particles $A_{1}$, $A_{2}, \cdots, A_{m}(m \geq 2)$ during the time $t_{1}$ where the correlation degree among these $m$ particles is totally $K_{1}=\mathrm{C}_{m}^{m}$; and then after time $t_{2}$, each of the particle $A_{i}$ $(i, j=1,2, \cdots, m)$ generates $m$ particles $m A_{i j}$ $(i, j=1,2, \cdots m)$ with $K_{2}=C_{m^{2}}^{m}, \cdots$, continuing to repeat to time $t_{n}$, there are $N_{n}^{m}=m^{n}$ particles from $A$ with $K_{n}=C_{m^{n}}^{m}$. Accordingly, the dimension $d$ of the regular network here is formed by the $N_{n}$ particles generated from particle $A$ during time $t_{n}$ :

$$
d=\lim _{n \rightarrow \infty} \frac{\ln K_{n}}{\ln N_{n}}=\lim _{n \rightarrow \infty} \frac{\ln C_{m^{n}}^{m}}{n \ln m}=m
$$

Proof: Mathematical induction is applied to solve this problem.

Firstly, we have an explanation, $d_{i}$ represents the dimensions of network space composed by $i^{n}$ particles.

(1) When $m$ is equal to 2 .

Through the first fission, there are 2 particles. Thus, the total correlation degree $K_{1}$ is $C_{2}^{2}$.

Through the second fission, there are $2^{2}$ particles. Thus, the total correlation degree $K_{2}$ is $C_{2^{2}}^{2}$.

Through the $n$ times fission, there will be $2^{n}$ particles. Thus, the total correlation degree $K_{n}$ is $C_{2^{n}}^{2}$.

$$
K_{n}=C_{2^{n}}^{2}=\frac{2^{n}\left(2^{n}-1\right)}{2}=2^{n-1}\left(2^{n}-1\right)
$$

(a)

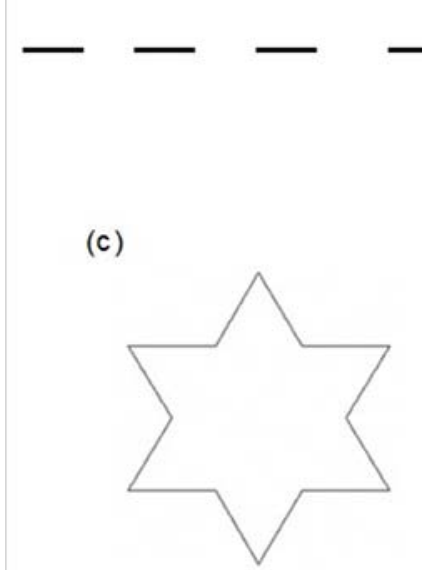

(b)

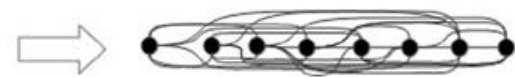

(d)

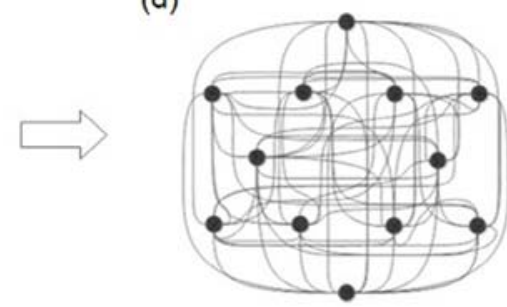

Figure 1. Fractal and complete-connective network graph. 
According to the Equation (2), we can attain:

$$
\begin{aligned}
d_{2} & =\lim _{n \rightarrow \infty} \frac{\ln K_{n}}{\ln N_{n}}=\lim _{n \rightarrow \infty} \frac{\ln \left[2^{n-1}\left(2^{n}-1\right)\right]}{\ln 2^{n}} \\
& =\lim _{n \rightarrow \infty} \frac{(n-1) \ln 2+\ln \left(2^{n}-1\right)}{n \ln 2}=2=m
\end{aligned}
$$

(2) When $m$ is equal to 3.

Through the first fission, there are 3 particles. Thus, the total correlation degree $K_{1}$ is $C_{3}^{3}$.

Through the second fission, there are $3^{2}$ particles. Thus, the total correlation degree $K_{2}$ is $C_{3^{2}}^{3}$.

Through the $n$ times fission, there will be $3^{n}$ particles. Thus, the total correlation degree $K_{n}$ is $C_{3^{n}}^{3}$.

$$
K_{n}=C_{3^{n}}^{3}=\frac{3^{n}\left(3^{n}-1\right)}{3}=3^{n-1}\left(3^{n}-1\right)\left(3^{n}-2\right)
$$

According to the Equation (2), we can attain:

$$
\begin{aligned}
d_{2} & =\lim _{n \rightarrow \infty} \frac{\ln K_{n}}{\ln N_{n}}=\lim _{n \rightarrow \infty} \frac{\ln \left[3^{n-1}\left(3^{n}-1\right)\left(3^{n}-2\right)\right]}{\ln 3^{n}} \\
& =\frac{(n-1) \ln 3+\ln \left(3^{n}-1\right)+\ln \left(3^{n}-2\right)}{n \ln 3}=3=m
\end{aligned}
$$

(3) For the same reason, when $m$ is equal to $k$. Through the $n$ times fission, the dimensions $d$ of network space composed by $k^{n}$ particles are equal to $k$.That's to say, $d_{k}=k$.

When $m=k+1$

$$
\text { Then } K_{n}=C_{(k+1)^{n}}^{(k+1)}, \quad N_{n}=(k+1)^{n}
$$

Firstly, we know when $n \rightarrow \infty$,

$$
K_{n} \stackrel{\text { a.s. }}{\longrightarrow}(k+1)^{n(k+1)-1}
$$

Therefore, we can attain:

$$
\begin{aligned}
d_{k+1} & =\lim _{n \rightarrow \infty} \frac{\ln K_{n}}{\ln N_{n}}=\lim _{n \rightarrow \infty} \frac{\ln C_{(k+1)^{n}}^{(k+1)}}{\ln (k+1)^{n}} \\
& =\lim _{n \rightarrow \infty} \frac{\ln C_{k^{n}}^{k} \cdot \ln k \cdot \ln C_{(k+1)^{n}}^{(k+1)}}{n \ln k \cdot \ln (k+1) \cdot \ln C_{k^{n}}^{k}} \\
& =k \cdot \lim _{n \rightarrow \infty} \frac{\ln k \cdot \ln C_{(k+1)^{n}}^{(k+1)}}{\ln (k+1) \cdot \ln C_{k^{n}}^{k}} \\
& =k \cdot \lim _{n \rightarrow \infty} \frac{\ln k \cdot[n(k+1)-1] \cdot \ln (k+1)}{\ln (k+1) \cdot(n k-1) \cdot \ln k} \\
& =k\left(1+\frac{1}{k}\right)=k+1=m
\end{aligned}
$$

We have got the conclusion that, through $n$ times fission, the dimension $d$ of the network space composed by the $m^{n}$ new particles is equal to $m$. The demonstration is completely shown in the Equation(2).

\section{Simple Calculation method of Dimension of Regular Network Based on the New De- finition of Dimension}

As predicted above, two new particles can be generated from only one particle, and each of the two new ones can generate extra two particles, so the correlation could be established among any two of these $2^{2}$ particles to form a complete-connective regular network(Figure 2-(a)). According to the Equation (2), the dimension of the new network here could be calculated as

$$
d=\frac{\ln C_{2^{2}}^{2}}{\ln 2}=\frac{\ln 6}{2 \ln 2} \approx 1.293 .
$$

Furthermore, if the newly generated $2^{2}$ particles get two more particles separately, the correlation could be established among any two of the $2^{3}$ particles, and the dimension of this complete-connective regular network could be calculated as $d=\frac{\ln C_{2^{3}}^{2}}{\ln 2^{3}}=\frac{2+\ln 7}{3 \ln 2} \approx 1.602$. To get correlation among the fresh $2^{4}$ particles split from those $2^{3}$ particles, then we can get another regular network with complete-connectivity, its dimension can b

calculated as $d=\frac{\ln C_{2^{4}}^{2}}{\ln 2^{4}}=\frac{2+\ln 7}{4 \ln 2} \approx 1.727 ; \cdots ;$ To put it briefly, the dimension of the complete-connective regular network will increase gradually, as the number of newly generated particles grows at a constant rate of 2 times. When the number of particles becomes $2^{n}$, and $n \rightarrow \infty$, the complete-connective regular network has the dimension $d=2$. (a)

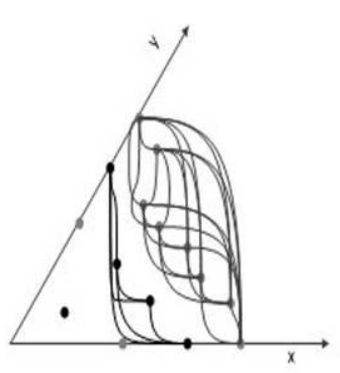

(b)

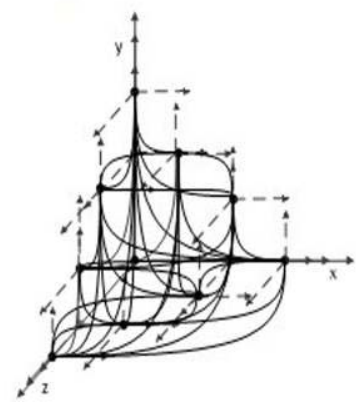

Figure 2. (a) shows Complete-connective regular networks built by particles growing at a constant rate of 2 times. (b) shows complete-connective regular networks built by particles growing at a constant rate of 3 times. 
To consider the situation that one particle can produce 3 new particles, and each of the 3 new ones can have other extra 3 particles, so totally there are $3^{2}$ particles and a complete-connective regular network based on the correlation among these particles (Figure 2-(b)). Also according to the definition of dimension, we can measure the network with dimension

$$
d=\frac{\ln C_{3^{2}}^{3}}{\ln 3^{2}}=\frac{2 \ln 2+\ln 21}{2 \ln 3} \approx 2.016 .
$$

Another network will be formed from the fission of the $3^{2}$, and the dimension of this network with totally $3^{3}$ particles will be calculated as

$$
d=\frac{\ln C_{3^{3}}^{3}}{\ln 3^{3}}=\frac{2(\ln 3+\ln 5)+\ln 13}{3 \ln 3} \approx 2.421 .
$$

If we continue, there will be the network composed by $3^{4}$ particles with dimension $d=\frac{\ln C_{3^{4}}^{3}}{\ln 3^{4}} \approx 2.584 ; \cdots ;$ In short, the dimension of the complete-connective regular network will increase gradually, as the number of newly generated particles grows at a constant rate of 3 times. When the number of particles becomes $3^{n}$ and $n \rightarrow \infty$, the complete-connective regular network has the dimension $d=3$.

\section{Conclusion}

Based on the Self-replication Space and the principle of the cantor set and the Koch curve, we establish a complete-connective regular network, and propose a new definition of the dimension due to the network correla- tion:

$$
d=\lim _{n \rightarrow \infty} \frac{\ln K}{\ln N} .
$$

We identify that the number of network nodes established by particles after fissions at a constant rate of $m$ times is $N_{n}=m^{n}$, and the original particle will not exist after the fission. Furthermore, our examination suggests that the total correlation degree among nodes is $K_{n}=C_{m^{n}}^{m}$, and as $n \rightarrow \infty$, the dimension $d=m$. Moreover, we also give a simplified method for the measurement of the dimension of the two regular networks. Then we can get a extension: the formation of the three-dimension Euclidean space may be built by the process of the Big Bang's continuously growing at a constant rate of 3 times, to some extent, this can provide more supportive explanation and judgment on the causal relationship between materials and space.

\section{REFERENCES}

[1] Song Chao-ming, Gallos L K, Havlin S,et al. How to calculate the fractal dimension of a complex network: The box covering algorithm [J]. Journal of Statistical Mechanics, 2007

[2] Song Chao-ming, Havlin S, Makse H A. Self-similarity of complex networks[J].Nature, 2005, 433: 392-395

[3] Song Chao-ming,Havlin S,Makse H A.Origins of fractality in the growth of complex networks[J].Nature Physics, 2006, 2: 275-281.

[4] Kim J S, Goh K-I, Kahng B, et al. A box-covering algorithm for fractal scaling in scale-free networks[J]. Chaos, 2007, 17 (2) 